\title{
Myocardial perfusion assessment by use of system identification method in a one-compartment model
}

\author{
Kocher M, Daire JL, Thévenaz P, Schindler T, Keller PF, Didier D, Vallée JP
}

\begin{abstract}
Cardiovascular magnetic resonance has been shown to provide high data quality for myocardial perfusion assessment. However, to analyze the perfusion data, some signal processing and modeling is needed to correct for motion related artifacts and limited spatial resolution. This study describes a method based on system identification, allowing, after a first step of image registration, to integrate and correct the partial volume effect in the myocardium perfusion quantification. This method is then applied to patients with coronary artery disease or hypertrophic obstructive cardiomyopathy.
\end{abstract}

\section{INTRODUCTION}

$\mathrm{M}$ YOCARDIAL perfusion assessment is an important issue in coronary artery disease in clinical practice. Time transit through a tissue can be estimated with a magnetic resonance (MR) first pass imaging technique and contrast media injection ${ }^{1-9}$. However, quantification of myocardial perfusion from the MRI remains a challenge. Compartmental model of myocardial perfusion have been proposed but are largely based on a manual drawing to extract the time transit curves from the organ of interest. The purpose of this study is to implement an automated image processing tool for the quantification of the myocardial perfusion from perfusion MRI. First, due to the respiratory motion of patient during acquisition, image registration is performed to accurately quantify the response of the myocardium to a blood contrast agent injection. Then, partial volume is corrected in mixed voxels containing both myocardium tissue and blood. This partial volume effect, has to be taken into account to avoid an overestimation of myocardial perfusion. Finally, this signal processing is applied to cardiac patients and the derived perfusion parameters are correlated to the results of thalium imaging or the coronary angiography.

Manuscript received April 2, 2007. This work was supported in part by the Center for Biomedical Imaging (CIBM) of the Geneva-Lausanne Universities and the EPFL, and the Swiss National Science Foundation (grant: PPOOB-68778)

Kocher M. and Thévenaz P. are with the Biomedical Imaging Group, EPFL, Station 17, 1015 Lausanne, Switzerland, (corresponding author: michel.kocher@hesge.ch).

Daire J.L., Didier D. and Vallée J.P. are with the Département d'imagerie et des sciences de l'information médicale, Geneva University Hospital, rue Micheli-du-Crest 24, 1211 Geneva, Switzerland.

Schindler T. and Keller P.F. are with the Department of Cardiology, Geneva University Hospital, rue Micheli-du-Crest 24, 1211 Geneva, Switzerland.

\section{MATERIAL AND METHODS}

\section{A. Patients}

Cardiac perfusion exams at rest were carried out in 10 cardiac patients ( 7 males, 3 females, mean age $59 \pm 13$ years) with an history of myocardial infarct. In 6 patients, the myocardial infarct was related to coronary artery disease and demonstrated on thalium scintigraphy. The remaining patients $(n=4)$ had a hypertrophic obstructive cardiomyopathy (HOCM) requesting a septal reduction by alcoolisation. This successful treatment that resulted in an effective myocardial infarct was attested during the coronary angiography. The HOCM patients were examined twice by MRI before and 2 days after treatment. An informed consent was obtained before each MR exam in agreement with the ethical rules of our institution.

\section{B. MRI Protocol}

Imaging was carried out on an Eclipse 1.5T MR system (Philips Medical Systems, Cleveland, OH) with an RFFAST sequence ${ }^{10}$ and following parameters: TI/TR/TE 28/3.74/1.5 ms, $50 \mathrm{kHz}$ bandwidth, flip angle $40^{\circ}, 90^{\circ}-180^{\circ}$ preparation pulses, and a $112 \times 128$ matrix, mean FOV $37 \mathrm{~cm}$ ( $\pm 2 \mathrm{~cm}$, according to patient's size). A cardiac surface coil and ECG trigger were used. Eight slices (4 short-axis, and 4 long-axis) were acquired during three to six cardiac cycles, depending on patient's heart rate, the average inter-image delay being 4 seconds $( \pm 0.5)$. A bolus of $0.08 \mathrm{mmol} / \mathrm{kg} \mathrm{Gd}$ DTPA was injected in a brachial vein followed by $10 \mathrm{ml}$ of isotonic saline with a Medrad ${ }^{\circledR}$ Spectris MR injector.

\section{Data analysis}

\section{1) Spatial registration}

The MRI heart acquisition is triggered based, which means that the acquisition instants are synchronized with the systole or with the diastole. Nevertheless, due to the respiration, the heart is spatially moving as a function of time. This movement has to be compensated in order to accurately compute the arterial input function $\operatorname{Cart}(t)$ and to register the pixels located in the myocardium to compute $\operatorname{Cmyo}(x, y, t)$.

Many two dimensional registration methods exist ${ }^{11,12}$. They differ in the metrics used to compare images together and in the geometric deformation they compensate. For this project, we have chosen the mutual information metrics instead of the least square one because of the contrast time variation in the image due to the contrast product flow in the 
heart. The chosen geometric deformation model is given by the following relation

$$
\left(\begin{array}{c}
x^{\prime} \\
y^{\prime} \\
1
\end{array}\right)=\left(\begin{array}{ccc}
z_{x} & s_{x} & d x \\
s_{y} & z_{y} & d y \\
0 & 0 & 1
\end{array}\right)\left(\begin{array}{l}
x \\
y \\
1
\end{array}\right)
$$

In this relation $\langle x, y\rangle$ and $\left\langle x, y^{\prime}\right\rangle$ are the pixel coordinates before and after transformation respectively. The affine transform parameters $z_{x}, z_{y}$ and $s_{x}, s_{y}$ describe the zoom and skewness factors respectively. These parameters are iteratively computed by an optimization process based on the Levenberg-Marquardt procedure.

\section{2) Estimation of the arterial input function}

The arterial signal $\operatorname{Cart}(t)$ is computed by applying a spatial average filter to all the $\langle x, y\rangle$ pixels belonging to the cavity's ROI. Considering a system identification approach which consists in applying an input signal to a linear known system and observing the output signal in order to estimate the model's parameters, $\operatorname{Cart}(t)$ is the single input signal whereas $\operatorname{Cmyo}(x, y, t)$ are the multiple output signals. This System Identification Single Input Multiple Outputs (SIMO) approach allows us to compute, for each pixel located in the myocardium, a set of parameters which locally describe the perfusion process.

\section{3) Compartmental perfusion model}

In this work, two perfusion models will be investigated, a single compartment model with and without partial volume. These two models are linear and are described by simple differential equations with constant coefficients.

The one compartment model describing the myocardium includes capillaries, interstitium, and cells, as a single compartment ${ }^{1}$. This model is well suited to describe the extravascular diffusion of Gd-DTPA, which has no cellular distribution and no metabolism ${ }^{13}$. Two parameters were used for the fit, $K 1$ and $K 2$, the first-order transfer constants from the left ventricular blood to the myocardium and from the myocardium back to the veins (in milliliters per minute per gram), according to the following equation and a tissue density of $1.04 \mathrm{~g}$ tissue $/ \mathrm{ml}$ tissue [10]:

$$
\dot{C}_{\text {myo }}(t)=K_{1} C_{\text {art }}(t)-K_{2} C_{\text {myo }}(t)
$$

where $\operatorname{Cmyo}(t)$ is the relative signal intensity in the myocardial compartment and $\operatorname{Cart}(t)$ the relative signal intensity in the blood of the left ventricle. The solution of Eq (2) has already been described for myocardial perfusion quantification by MRI ${ }^{5,14}$. The fraction volume of distribution of Gd-DTPA in the myocardium is defined as:

$$
V d=\frac{K 1}{K 2}(1-H c t)
$$

$H c t$ being the hematocrit value (set to a constant value of $45 \%)$.

In the Laplace domain, the equation (2) corresponds to the following transfer function containing 1 real pole :

$$
H(s)=\frac{K_{1} / K_{2}}{1+s / K_{2}}
$$

The following bloc diagram describes this relation (Fig. $1)$ :

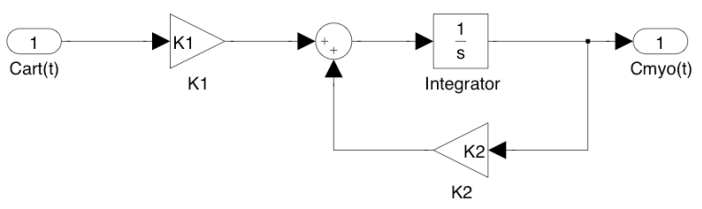

Fig.

1: This block diagram describes a one compartmental model without partial volume.

This model is accurate if the considered myocardium pixels are not located too close to the cavity because of the effect of the partial volume which represent the combined influence of the cavity and the myocardium. A better model, which takes into account the partial volume is described by the following equation.

$$
C_{\text {myopV }}=\alpha C_{\text {myo }}+(1-\alpha) C_{\text {art }}
$$

In the Laplace domain, the equation (5) corresponds to the following transfer function containing one real pole and one real zero :

$$
H_{P V}(s)=K \frac{1+T_{z} s}{1+T_{p} s}
$$

The following bloc diagram describes this relation (Fig. 2):

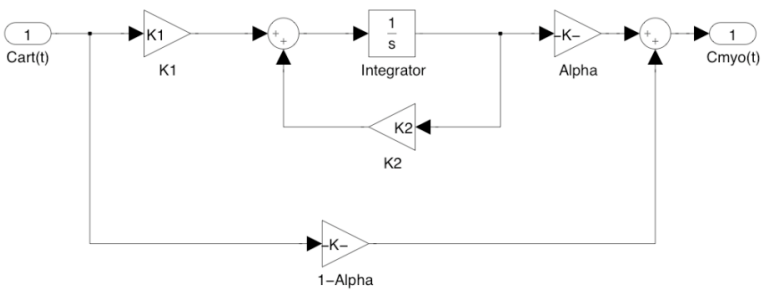

Fig. 2: This block diagram describes a one compartemental model with partial volume. The amount of partial volume is described by the alpha parameter in the range from 0 to 1 (no partial volume).

For each of these models, the unknown parameters are estimated by minimizing $J$ which is the norm of the difference between the real $\operatorname{Cmyo}(x, y, t)$ data and the estimated one. This estimation is performed by using an optimization approach ${ }^{15}$.

\section{RESULTS}

1) Spatial registration

The figure 3 illustrates the noise cleaning effect of the registration process. It shows the standard deviation value computed at each time on a one pixel wide ring belonging to the myocardium.

Applied on the population of this study, image registration allowed to decrease significantly signal variation $\sigma$ from 55.3 to $45.4(\mathrm{p}<0.001)$. 

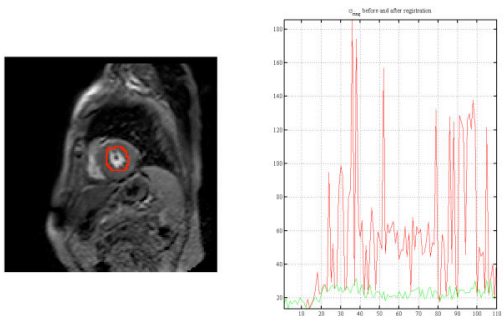

Fig. 3: Representative example of registration: signal variations along a ring placed in the myocardium is well decreased after registration allowing a correct pixel by pixel analysis on all acquired dynamics.

2) Model parameters identification

In the case of the model without partial volume consideration, figure 4 describes the objective function, $J(K 1, K 2)$, both for the normal and infarcted case.

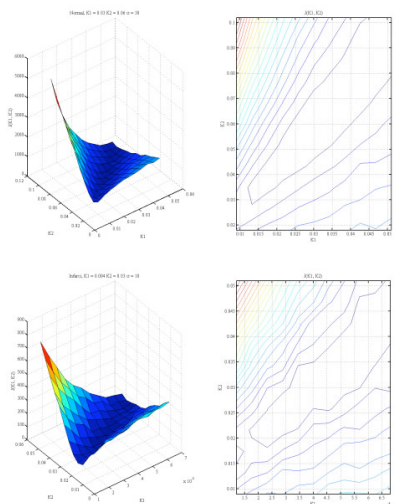

Fig. 4: 3D and contour level view of the performance surface $J(K 1, K 2)$ in a normal case (up) and infarcted case (bottom).

It comes out that the minimum of $J(K 1, K 2)$, which we are looking for, is not a point but rather a line defined by $K 1 / K 2=$ constant. This implies that the ratio $K 1 / K 2$ will be correctly estimated but that each $K 1$ and $K 2$ can suffer a bias.

In the case of the second model, with partial volume, figure 5 describe $J(K 1, K 2, \alpha)$, both for the normal and infarcted case.

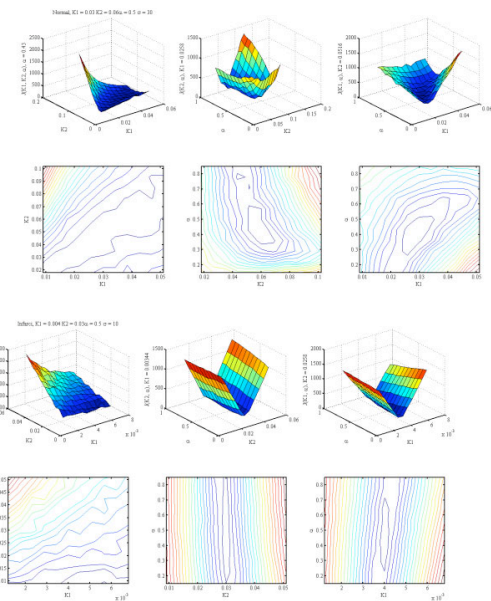

Fig. 5: 3D and contour level view of the performance surface $J_{P V}(K 1, K 2, \alpha)$ in a normal case (up) and infarcted case (bottom) .
It comes out that the function $J_{P V}(K 1, K 2, \alpha)$ has better minimum searching features than the function $J(K 1, K 2)$ allowing a more precise estimation of $K 1$ and $K 2$ in the presence of noise.

Figure 6 shows that, for a $\operatorname{Cmyo}(x, y, t)$ having only $10 \%$ $(\alpha=0.9)$ of partial volume, the advantage of using the second model. Indeed, the first model overestimates $K 1$ and $K 2$ by approximately $80 \%$ whereas, when partial volume is corrected, the obtained values of $K 1$ and $K 2$ are very close to the theoretical values.
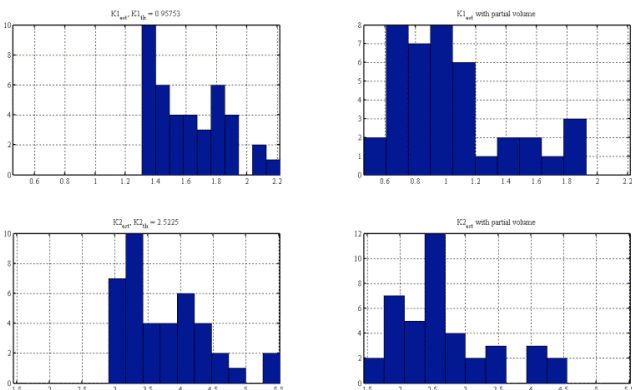

Fig. 6: Simulation results : from an arterial input and initial fixed K1 and $\mathrm{K} 2$ values with different noise, 20 realizations of the myocardium response are simulated. The relative difference between the theoretical value and the estimated without partial volume are shown (left) and the one with partial volume correction (right) (first row: $K 1,2^{\text {nd }} \mathrm{rw}$ : $K 2$ ). The model with partial volume leads to both a better estimation of each parameter and a smaller $J$ value.

\section{3) Application to patients}

According to the SPECT study and the coronary angiography, 27 segments were infarcted and 123 were normal. Without partial volume consideration, the $K 1$ perfusion value in the normal region was $0.970 \pm 0.246$ $\mathrm{ml} / \mathrm{min} / \mathrm{g}$ and $0.493 \pm 0.196 \mathrm{ml} / \mathrm{min} / \mathrm{g}$ in the infarcted sectors. Taking into account the partial volume effect, the $K 1$ perfusion value in the normal region was $0.912 \pm 0.254$ $\mathrm{ml} / \mathrm{min} / \mathrm{g}$ and $0.472 \pm 0.186 \mathrm{ml} / \mathrm{min} / \mathrm{g}$ in the infarcted sectors. In both models, infarcted sector exhibited significantly lower perfusion values than normal regions $(\mathrm{p}<0.0001)$ as shown on figure $7 . K 2$ values exhibited also a significant decrease between normal $(2.47 \pm 0.80 \mathrm{ml} / \mathrm{min} / \mathrm{g})$ and infarcted $(1.39 \pm 0.59 \mathrm{ml} / \mathrm{min} / \mathrm{g})$ sectors $(\mathrm{p}<0.0001)$. With partial volume correction, about the same difference is observed $(2.45 \pm 0.88 \mathrm{ml} / \mathrm{min} / \mathrm{g}$ vs. $1.74 \pm 1.80 \mathrm{ml} / \mathrm{min} / \mathrm{g}$, $\mathrm{p}<0.01)$ No differences were found in the fractional volume of distribution $V d$ of the tracer between normal $(21.5 \pm$ $4.9 \%)$ and infarcted region $(19.7 \pm 6.9 \%)(p=0.064)$.

On normal sectors, the average partial volume fraction (1$\alpha)$ was about $2.2 \% \quad(\alpha=0.9788, \quad(\min : 0.8064, \max : 1.0)$ whereas in infarcted sectors $(1-\alpha)$ is about $2.78 \%$ ( $\alpha=0.9722$, min:0.9152, max:1.0). Consequently, significant differences were found in $K 1$ values and distribution volumes in values with and without partial volume correction $(\mathrm{p}<0.001)$ (fig. 8). 


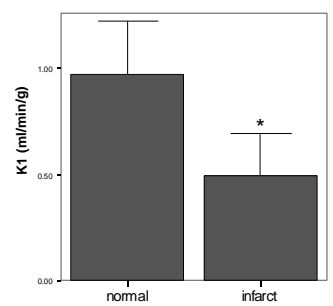

Fig. 7: Myocardial perfusion value $K 1$ is significantly lower in infarcted sector compared to the normal regions in case of partial volume correction $(*: \mathrm{p}<0.0001)$

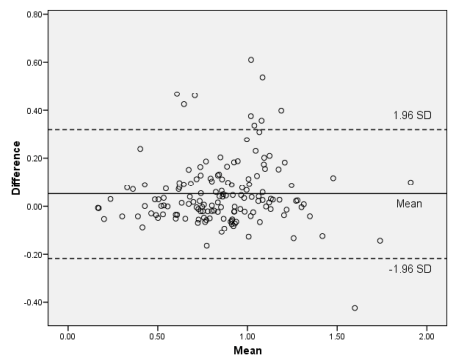

Fig. 8: A Bland-Altman analysis shows a bias of $5.85 \%$ in perfusion value due to the correction of the partial volume effect.

For the HOCM patients, the perfusion results of the second MR exam demonstrate the successful treatment of septal reduction by alcoolisation. A clear deficit of perfusion was observed in all cases in the septal region as shown on figures 9 and 10 .

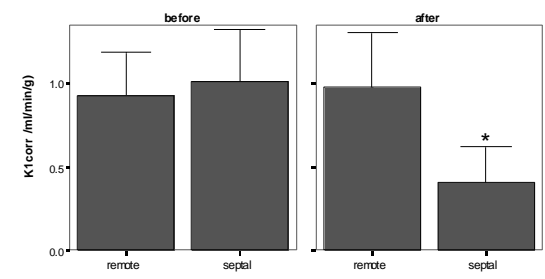

Fig. 9: $K 1$ values in HOCM patients in septal and remote regions before and after treatment: a significant decrease is observed in the septal region $(*: \mathrm{p}<0.01)$

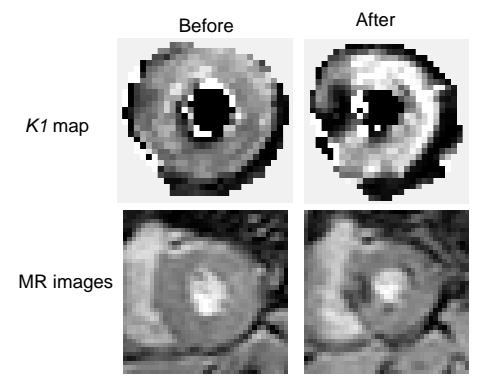

Fig. 10: Representative perfusion MR images and the corresponding $K 1$ maps. In this case of HOCM patients, a clear perfusion deficit is observed in septal sector.

\section{DISCUSSION}

This study demonstrates the use and the importance of signal processing and system identification methods in cardiac perfusion measurement. Since MR images are acquired in a short axis view at the same phase of the cardiac cycle, we need to correct the displacement of the myocardium related to the respiratory movement to guarantee an accurate pixel by pixel analysis for all dynamics. The choice of a method based on the mutual information metrics is well appropriate in cardiac MRI where the first pass of the contrast media ensures a high contrast between myocardium and left ventricle cavity.

Partial volume effect is a critical parameter of the method and has to be taken into account in the model. Indeed, myocardium voxel contamination by the arterial input function increases the response of the myocardium and consequently overestimates the perfusion value $K 1$. The observed partial volume in myocardium can be explained by an insufficient spatial resolution, slices not exactly perpendicular to the myocardium, or by heart movement due to contraction and/or breathing. In normal regions, we observed about less than $3 \%$ of myocardium contamination inducing a change of $5 \%$ in $K 1$ value, which is consistent with other study ${ }^{16}$. The clinical application of this protocol gave perfusion value in agreement with previous studies and demonstrated that reliable parametric maps of the myocardial perfusion can be obtained.

\section{REFERENCES}

[1] Vallee JP. MRI quantitative myocardial perfusion with compartmental analysis: a rest and stress study. Magn Reson Med 1997;38(6):981-9.

[2] Vallee JP. Quantification of myocardial perfusion by MRI after coronary occlusion. Magn Reson Med 1998;40(2):287-97.

[3] Vallee JP. Quantification of myocardial perfusion with FAST sequence and Gd bolus in patients with normal cardiac function. J Magn Reson Imaging 1999;9(2):197-203.

[4] Vallee JP. Absolute renal blood flow quantification by dynamic MRI and Gd-DTPA. Eur Radiol 2000;10(8):1245-52.

[5] Larsson HB. In vivo quantification of the unidirectional influx constant for Gd-DTPA diffusion across the myocardial capillaries with MR imaging. J Magn Reson Imaging 1994;4(3):433-40.

[6] Larsson HB. Myocardial perfusion modeling using MRI. Magn Reson Med 1996;35(5):716-26.

[7] Wilke N. Myocardial perfusion reserve: assessment with multisection, quantitative, first-pass MR imaging. Radiology 1997;204(2):373-84.

[8] Wilke NM. Magnetic resonance first-pass myocardial perfusion imaging: clinical validation and future applications. J Magn Reson Imaging 1999;10(5):676-85.

[9] Epstein FH. Multislice first-pass cardiac perfusion MRI: validation in a model of myocardial infarction. Magn Reson Med 2002;47(3):482-91.

[10] Gyngell ML. The application of steady-state free precession in rapid 2DFT NMR imaging: FAST and CE-FAST sequences. Magn Reson Imaging 1988;6(4):415-9.

[11] Thevenaz P. Interpolation revisited. IEEE Trans Med Imaging 2000;19(7):739-58.

[12] Thévenaz P UM. Optimization of mutual information for multiresolution image registration. IEEE Trans Image Processing 2000;9(12):2083.

[13] Weinmann HJ. Characteristics of gadolinium-DTPA complex: a potential NMR contrast agent. AJR Am J Roentgenol 1984;142(3):619-24.

[14] Diesbourg LD. Quantification of myocardial blood flow and extracellular volumes using a bolus injection of Gd-DTPA: kinetic modeling in canine ischemic disease. Magn Reson Med 1992;23(2):239-53.

[15] Ljoung L. System Identification - Theory For The User. PTR Prentice Hall, Upper Saddle River ed. NJ; 1999.

[16] Kostler H. Correction for partial volume errors in MR heart perfusion imaging. Magn Reson Med 2004;51(4):848-52. 